\title{
CHECK LIST OF THE MELASTOMATACEAE OF EQUATORIAL GUINEA
}

\author{
by \\ INGRID PARMENTIER \& DANIEL GEERINCK \\ Laboratoire de Botanique Systématique et de Phytosociologie. \\ CP169, Université Libre de Bruxelles, 50 av. F.D. Roosevelt, 1050 Brussels \\ <inparmen@ulb.ac.be>
}

\begin{abstract}
Resumen
Parmentier, I. \& D. GeERINCK (2003). Catálogo de Melastomataceae de Guinea Ecuatorial. Anales Jard. Bot. Madrid 60(2): 331-346 (en inglés).

Se presenta el catálogo florístico de la familia Melastomataceae en Guinea Ecuatorial. Se recogen un total de 57 táxones. Tres especies fueron aceptadas teniendo en cuenta solamente la literatura. Su distribución sugiere que su presencia en Guinea Ecuatorial es muy probable. En Annobón están presentes 6 especies, 23 en Bioko y 49 en Río Muni. Los géneros mejor representados son Memecylon (10 especies), Calvoa (10) y Tristemma (7).Veintiséis táxones son citados por primera vez en Guinea Ecuatorial. Se propone Heterotis obamae Lejoly \& Lisowski como sinónimo de Heterotis arenaria Jacq.-Fél.

Palabras clave: Guinea Ecuatorial, Melastomataceae, Heterotis obamae, Heterotis arenaria, check list, Annobón, Río Muni, Bioko.
\end{abstract}

\begin{abstract}
Parmentier, I. \& D. Geerinck (2003). Check list of the Melastomataceae of Equatorial Guinea. Anales Jard. Bot. Madrid 60(2): 331-346.

A chec klist of the Melastomataceae of Equatorial Guinea is presented with 57 taxa. Three species were accepted based only on literature records, their distribution area strongly suggests their presence in Equatorial Guinea. Six species are known from Annobón, 23 from Bioko and 49 from Río Muni. Best-represented genera are Memecylon (10), Calvoa (10) and Tristemma (7). Twenty-six taxa are newly recorded for the country. Heterotis abamae Lejoly \& Lisowski is set in synonymy with the previously described Heterotis arenaria Jacq.-Fel.

Key words: Equatorial Guinea, Melastomataceae, Heterotis obamae, Heterotis arenaria, check list, Annobón, Río Muni, Bioko.
\end{abstract}

\section{INTRODUCTION}

Equatorial Guinea is located in Western Central Africa (fig. 1). The country includes two main islands in the Gulf of Guinea: Bioko $\left(2017 \mathrm{~km}^{2}\right), 32 \mathrm{~km}$ from the coast of Cameroon, and Annobón $\left(17 \mathrm{~km}^{2}\right), 400 \mathrm{~km}$ from Gabon. The continental mainland, Río Muni $\left(26000 \mathrm{~km}^{2}\right)$ is located between $1^{\circ}$ and $2^{\circ} 30^{\prime} \mathrm{N}$ and $9^{\circ} 30^{\prime}$ and $11^{\circ} 30^{\prime} \mathrm{E}$. Elobey and
Corisco are small islands near the border of Río Muni with Gabon. Pico Basile, on Bioko Island, is the highest peak (altitude: $3011 \mathrm{~m}$ ) and occupies half of the island, the other half being occupied by two other volcanoes: La caldera de Luba (2261 m) and Pico Biaó (2009 m) (Pérez DeL VAL, 1996). Malabo, north of the island, has a drier season from December to January and annual precipitation of $1932 \mathrm{~mm}$ per year. Pluviometry is more 
important in the southern part of the island, with up to $10150 \mathrm{~mm}$ annual rainfall at Ureca. The temperature oscillates around $26{ }^{\circ} \mathrm{C}$ at sea level. There are strong variations in precipitation, temperature and humidity with al-

titude (Pérez del Val, 1996). From West to East, Río Muni is divided into three mains parts. The coastal zone includes a littoral sandy plain about $30 \mathrm{~km}$ wide followed by small hills about $200 \mathrm{~m}$ high. The Niefang
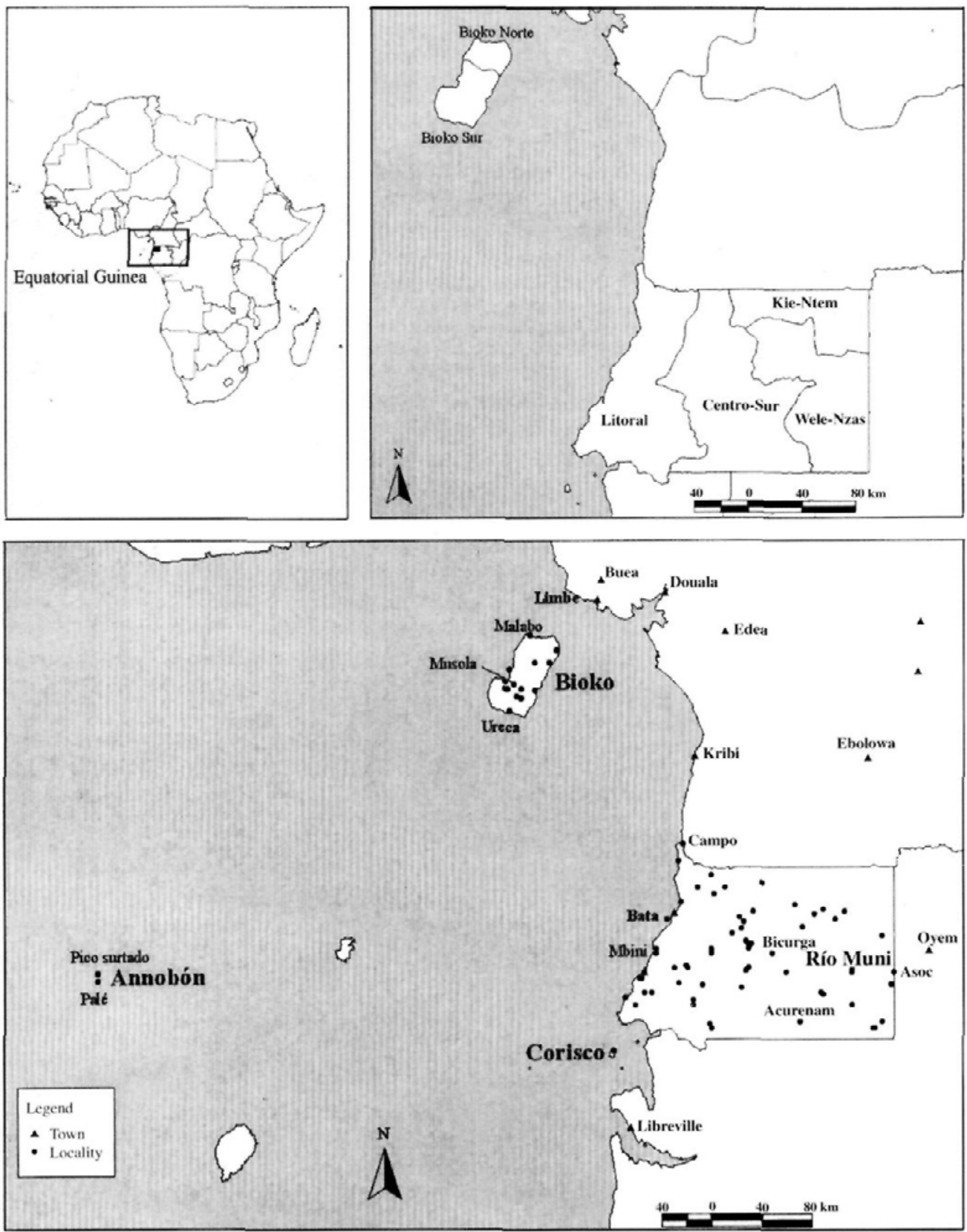

Fig. 1.-Equatorial Guinea in Africa, provinces, and localities of Melastomataceae collections. 
Mountain Range reaching $1200 \mathrm{~m}$ crosses Río Muni. The eastern part of the country is a vast plateau of 600 to 700 m altitude scattered with inselbergs. Climate in Río Muni is equatorial with two drier seasons: from July to September and from December to February. Mean precipitation $2500 \mathrm{~mm} /$ year, temperature is around $26^{\circ} \mathrm{C}$ all year.

Melastomataceae collections from Equatorial Guinea in the last twenty years were mainly collected by the Real Jardín Botánico of Madrid and the Laboratoire de Botanique systématique et de Phytosociologie of the Université Libre de Bruxelles. A good review of the botanical work in Equatorial Guinea is given in Velayos \& AEDo (2001). Most recent collections from Río Muni in BRLU were collected by Senterre for his study of the forest of the Parque Nacional de Monte Alén and the Parque Nacional de Nsork (SENTERre \& NGuema, 2001; Senterre, 2001) and by Parmentier, with her studies on the inselberg vegetation (PARMENTIER \& al., 2001; Parmentier \& NGUema, 2001; PARMeNTIER, 2002).

Early general studies giving information on Melastomataceae of West tropical Africa come from Hurchinson (1928), GUINEA (1946, 1951) and HuTCHINSON \& DALZIEL (1954). The major work of Jacques-Félix, is our main reference for this paper, especially his flora of Melastomataceae from Cameroon (JACQUES-FÉLIX, 1983a) and Gabon (JACQUESFéLIX, 1983b). It was usefully completed by a recent revision of Calvoa (FIGUEIREDO, 2001). Recent works notifying the occurrence of Melastomataceae species on Bioko are those of MORALES $(1994,1995)$ and FERNÁNDEZ CASAS \& Morales ValVerde (1995).

\section{MATERIALS AND METHODS}

The check list is mainly based on the examination of about 600 Melastomataceae herbarium specimens from BATA (Herbario Nacional de Guinea Ecuatorial), BRLU (Herbarium de l'Université Libre de Bruxelles) and MA (Herbario del Real Jardín Botánico de Madrid). Determinations were based on the works of Jacques-Félix, especially in the flora of Melastomataceae of Gabon (JACQUES-FÉlIX, 1983a) and Cameroon (JACQUES-FÉLIX, 1983b) completed by more recent works (BORHIDI, 1993; JACQUES-FÉLIX, 1987; J. LEJOLY \& S. LISOWSKI, 1999c; FIGUEIREDO, 2001). Bibliographic records from Melastomataceae in Equatorial Guinea were obtained mainly from AEDO \& al. (1999), completed by recent publications like those of LEJOLY \& LISOWSKI (1999a,b,c), Parmentier \& NGuema (2001), PARMentier \& al. (2001), PARMENTIER (2002). Doubtful records and insufficiently known species are given at the end of the check list. The locality names used in this check list have been brought up-to-date and made uniform (table 1), localities are mapped in figure 1. Those localities are in agreement with those cited in the check list of the Pteridophytes of Equatorial Guinea (VELAYOS \& al., 2001). The check list is alphabetically ordered by species within genera. Under each accepted taxon, the accepted synonyms used in the literature of Equatorial Guinea are included (JACQUES-FÉLIX, 1974b; LEBRUN \& STORK, 1991, 1995, 1997; FIGUEIREDO, 2001). Specimens are alphabetically arranged within province, locality and collector. The herbaria where they are deposited are mentioned. Herbarium codes for MA specimens are given. The indice "st" means that a specimen is sterile. Previous bibliographical records for each taxon are listed. When the taxon was recorded under an accepted synonym it is indicated (i.e. LEJOLY, 1999b: 118, sub Dissotis invingiana).

New signalisations for the country are indicated by a "*" before the taxon name.

\section{THE CHECK LIST}

\section{Amphiblemma mildbraedii Gilg ex Engl.}

Bioкo Sur: Moca, Fernández Casas 11486 (MA 555825), Guinea 1968 (MA 268681). Moca-Riaba, por el camino viejo, Fernández Casas 11846 (MA 555826). Malabo-Moca, km 65-66, Carvalho 3767 (MA 568051); ibidem, km 67. Carvalho 2484 (MA 558405); ibidem, km 70, Carvalho 2617 (555824). Musola, Guinea 1723 (MA 384342), 1724 (MA 384344). 


\section{TABLE 1}

LOCALITIES OF MELASTOMATACEAE COLLECTIONS IN EQUATORIAL GUINEA WITH GEOGRAPHICAL COORDINATES

\begin{tabular}{|c|c|c|c|}
\hline Acasakira, rocher (Wele-Nzas) & $1^{\circ} 7^{\prime} \mathrm{N}-11^{\circ} 15^{\prime} \mathrm{E}$ & Moca (Bioko Sur) & $3^{\circ} 20^{\prime} \mathrm{N}-8^{\circ} 40^{\prime} \mathrm{E}$ \\
\hline Acurenam (Centro-Sur) & $1^{\circ} 7^{\prime} \mathrm{N}-10^{\circ} 40^{\prime} \mathrm{E}$ & Mongola (Wele-Nzas) & $1^{\circ} 46^{\prime} \mathrm{N}-10^{\circ} 41^{\prime} \mathrm{E}$ \\
\hline $\begin{array}{l}\text { Akoak Ebanga, inselberg } \\
\text { (Wele-Nzas) }\end{array}$ & $1^{\circ} 4^{\prime} \mathrm{N}-11^{\circ} 12^{\prime} \mathrm{E}$ & $\begin{array}{l}\text { Monte Alén (Centro-Sur) } \\
\text { Monte Mitra (Centro-Sur) }\end{array}$ & $\begin{array}{r}1^{\circ} 37^{\prime} \mathrm{N}-10^{\circ} 19^{\prime} \mathrm{E} \\
1^{\circ} 21^{\prime} \mathrm{N}-9^{\circ} 75^{\prime} \mathrm{E}\end{array}$ \\
\hline Akuom, inselberg (Wele-Nzas) & $1^{\circ} 49^{\prime} \mathrm{N}-10^{\circ} 55^{\prime} \mathrm{E}$ & Mungum, inselberg (Wele-Nzas) & $1^{\circ} 19^{\prime} \mathrm{N}-10^{\circ} 49^{\prime} \mathrm{E}$ \\
\hline Andom, inselberg (Wele-Nzas) & $1^{\circ} 27^{\prime} \mathrm{N}-11^{\circ} 2^{\prime} \mathrm{E}$ & Musola (Bioko Sur) & $3^{\circ} 26^{\prime} \mathrm{N}-8^{\circ} 37^{\prime} \mathrm{E}$ \\
\hline Añisok (Wele-Nzas) & $1^{\circ} 51^{\prime} \mathrm{N}-10^{\circ} 46^{\prime} \mathrm{E}$ & Ncoho (Litoral) & $1^{\circ} 14^{\prime} \mathrm{N}-9^{\circ} 54^{\prime} \mathrm{E}$ \\
\hline Arenas Blancas, playa & $3^{\circ} 32^{\prime} \mathrm{N}-8^{\circ} 35^{\prime} \mathrm{E}$ & Ngoma (Litoral) & $1^{\circ} 14^{\prime} \mathrm{N}-9^{\circ} 29^{\prime} \mathrm{E}$ \\
\hline $\begin{array}{l}\text { (Bioko Sur) } \\
\text { Asoc, inselberg (Wele-Nzas) }\end{array}$ & $1^{\circ} 27^{\prime} \mathrm{N}-11^{\circ} 20^{\prime} \mathrm{E}$ & $\begin{array}{l}\text { Ngong Mocomo (Wele-Nzas) } \\
\text { Niefang (Centro-Sur) }\end{array}$ & $\begin{array}{r}1^{\circ} 4^{\prime} \mathrm{N}-11^{\circ} 11^{\prime} \mathrm{E} \\
1^{\circ} 50^{\prime} \mathrm{N}-10^{\circ} 14^{\prime} \mathrm{E}\end{array}$ \\
\hline Ayamiken (Litoral) & $2^{\circ} 7^{\prime} \mathrm{N}-10^{\circ} 2^{\prime} \mathrm{E}$ & Nsum Esangüi (Wele-Nzas) & $1^{\circ} 42^{\prime} \mathrm{N}-11^{\circ} 15^{\prime} \mathrm{E}$ \\
\hline Ayene (Wele-Nzas) & $1^{\circ} 55^{\prime} \mathrm{N}-10^{\circ} 38^{\prime} \mathrm{E}$ & Obamico (Parque N. de Nsork) & $1^{\circ} 14^{\prime} \mathrm{N}-11^{\circ} 2^{\prime} \mathrm{E}$ \\
\hline Bahía de Venus (Bioko Norte) & $3^{\circ} 46^{\prime} \mathrm{N}-8^{\circ} 44^{\prime} \mathrm{E}$ & Oyac Esom (Centro-Sur) & $1^{\circ} 53^{\prime} \mathrm{N}-10^{\circ} 50^{\prime} \mathrm{E}$ \\
\hline Baríobe (Bioko Norte) & $3^{\circ} 35^{\circ} \mathrm{N}-8^{\circ} 52^{\prime} \mathrm{E}$ & Palé (Annobon) & $1^{\circ} 24^{\prime} \mathrm{N}-5^{\circ} 38^{\prime} \mathrm{E}$ \\
\hline $\begin{array}{l}\text { Belebú Balachá de Riaba } \\
\text { (Bioko Sur) }\end{array}$ & $3^{\circ} 24^{\prime} \mathrm{N}-8^{\circ} 34^{\prime} \mathrm{E}$ & $\begin{array}{l}\text { Parque Nacional de Monte Alén, } \\
2 \mathrm{~km} \text { au NE du site de traversée }\end{array}$ & $1^{\circ} 62^{\prime} \mathrm{N}-10^{\circ} 8^{\prime} \mathrm{E}$ \\
\hline Biaó, lago (Bioko Sur) & $3^{\circ} 21^{\prime} \mathrm{N}-8^{\circ} 38^{\prime} \mathrm{E}$ & du Río pour aller aux cataractas & \\
\hline Bicaba (Centro-Sur) & $1^{\circ} 48^{\prime} \mathrm{N}-10^{\circ} 16^{\prime} \mathrm{E}$ & (Centro-Sur) & \\
\hline Bicurga, inselberg (Centro-Sur) & $1^{\circ} 35^{\prime} \mathrm{N}-10^{\circ} 28^{\prime} \mathrm{E}$ & Parque Nacional de Monte Alén, & $1^{\circ} 45^{\prime} \mathrm{N}-10^{\circ} 15^{\prime} \mathrm{E}$ \\
\hline Bolondo (Litoral) & $1^{\circ} 37^{\prime} \mathrm{N}-9^{\circ} 38^{\prime} \mathrm{E}$ & $2 \mathrm{~km}$ au NO du transect de Mont & \\
\hline Bome (Litoral) & $1^{\circ} 49^{\prime} \mathrm{N}-9^{\circ} 43^{\circ} \mathrm{E}$ & Chocolate (Centro-Sur) & \\
\hline $\begin{array}{l}\text { Campo, desembocadura del río } \\
\text { (Litoral) }\end{array}$ & $2^{\circ} 20^{\prime} \mathrm{N}-9^{\circ} 50^{\prime} \mathrm{E}$ & $\begin{array}{l}\text { Parque Nacional de Monte Alén, } \\
\text { à } 250 \mathrm{~m} 60^{\circ} \mathrm{NE} \text { du transect de }\end{array}$ & $1^{\circ} 6$ 'N-10 $1^{\circ} \mathrm{E}$ \\
\hline Cogo (Litoral) & $1^{\circ} 5^{\prime} N-9^{\circ} 42^{\prime} E$ & Mosumo (Centro-Sur) & \\
\hline Corisco, isla (Litoral) & $0^{\circ} 55^{\prime} \mathrm{N}-9^{\circ} 20^{\prime} \mathrm{E}$ & Parque Nacional de Monte Alén, & $1^{\circ} 37^{\prime} \mathrm{N}-10^{\circ} 18^{\prime} \mathrm{E}$ \\
\hline Cupapa (Bioko Norte) & $3^{\circ} 40^{\prime} \mathrm{N}-8^{\circ} 55^{\prime} \mathrm{E}$ & dalle rocheuse d'Engong & \\
\hline Dumu, inselberg (Wele-Nzas) & $1^{\circ} 22^{\prime} \mathrm{N}-11^{\circ} 19^{\prime} \mathrm{E}$ & (Centro-Sur) & \\
\hline Ecucu (Litoral) & $1^{\circ} 56^{\prime} \mathrm{N}-9^{\circ} 49^{\prime} \mathrm{E}$ & Parque Nacional de Monte Alén, & $1^{\circ} 40^{\prime} \mathrm{N}-10^{\circ} 17^{\prime} \mathrm{E}$ \\
\hline Elendé (Litoral) & $2^{\circ} 13^{\prime} \mathrm{N}-9^{\circ} 48^{\prime} \mathrm{E}$ & dalle rocheuse de Monte Alén & \\
\hline Emangós (Litoral) & $1^{\circ} 23^{\prime} \mathrm{N}-9^{\circ} 48^{\prime} \mathrm{E}$ & (Centro-Sur) & \\
\hline Enkumekie (Centro-Sur) & $1^{\circ} 43^{\prime} \mathrm{N}-10^{\circ} 11^{\prime} \mathrm{E}$ & Parque Nacional de Monte Alén, & $1^{\circ} 36^{\prime} \mathrm{N}-10^{\circ} 2^{\prime} \mathrm{E}$ \\
\hline Etembue (Litoral) & $1^{\circ} 17^{\prime} \mathrm{N}-9^{\circ} 25^{\prime} \mathrm{E}$ & entre la station Ecofac de Mosum & \\
\hline Evinayong (Centro-Sur) & $1^{\circ} 27^{\prime} \mathrm{N}-10^{\circ} 34^{\prime} \mathrm{E}$ & et Monte Boracho & \\
\hline Handje (Litoral) & $1^{\circ} 25^{\prime} \mathrm{N}-9^{\circ} 31^{\prime} \mathrm{E}$ & Parque Nacional de Monte Alén, & $1^{\circ} 52^{\prime} \mathrm{N}-10^{\circ} 20^{\prime} \mathrm{E}$ \\
\hline Jandje (Litoral) & $1^{\circ} 27^{\prime} \mathrm{N}-9^{\circ} 33^{\prime} \mathrm{E}$ & près de la Cabaña de Esamalan & \\
\hline Kukumankok (Wele-Nzas) & $1^{\circ} 18^{\prime} \mathrm{N}-10^{\circ} 50^{\prime} \mathrm{E}$ & (Centro-Sur) & \\
\hline Laña, cascada del río (Centro-Sur) & $1^{\circ} 35^{\prime} \mathrm{N}-10^{\circ} 2^{\prime} \mathrm{E}$ & Parque Nacional de Monte Alén, & $1^{\circ} 4^{\prime} \mathrm{N}-10^{\circ} 2^{\prime} \mathrm{E}$ \\
\hline Loreto, lago (Bioko Sur) & $3^{\circ} 24^{\prime} \mathrm{N}-8^{\circ} 40^{\prime} \mathrm{E}$ & nrès de la Cahaña Ecofar de & \\
\hline Luba (Bioko Sur) & $3^{\circ} 27^{\prime}$ N-8 $33^{\prime} E$ & Misergue (Centro-Sur) & \\
\hline Malabo, aeropuerto (Bioko Norte) & $3^{\circ} 46^{\prime} \mathrm{N}-8^{\circ} 44^{\prime} \mathrm{E}$ & Parque Nacional de Monte Alén, & $1^{\circ} 64^{\prime} \mathrm{N}-10^{\circ} 24^{\prime} \mathrm{E}$ \\
\hline Mandjani (Litoral) & $1^{\circ} 19^{\prime} \mathrm{N}-10^{\circ} 49^{\prime} \mathrm{E}$ & sur la piste lago Atoc-Moca & \\
\hline Mayang (Litoral) & $1^{\circ} 29^{\prime} \mathrm{N}-9^{\circ} 52^{\prime} \mathrm{E}$ & (Centro-Sur) & \\
\hline Mbambala, río (Litoral) & $1^{\circ} 16^{\prime} \mathrm{N}-9^{\circ} 54^{\prime} \mathrm{E}$ & Parque Nacional de Monte Alén, & $1^{\circ} 39^{\prime} \mathrm{N}-10^{\circ} 18^{\prime} \mathrm{E}$ \\
\hline Mbini (Litoral) & $1^{\circ} 35^{\prime} N-9^{\circ} 38^{\prime} E$ & transect de Monte Alén & \\
\hline Mboete (Litoral) & $1^{\circ} 19^{\prime} \mathrm{N}-9^{\circ} 36^{\prime} \mathrm{E}$ & (Centro-Sur) & \\
\hline Mfuin, inselberg (Kie-Ntem) & $1^{\circ} 52^{\prime} \mathrm{N}-10^{\circ} 59^{\prime} \mathrm{E}$ & Parque Nacional de Monte Alén, & $1^{\circ} 39^{\prime} \mathrm{N}-10^{\circ} 19^{\prime} \mathrm{E}$ \\
\hline & $1^{\circ} 41^{\prime} \mathrm{N}-9^{\circ} 38^{\prime} \mathrm{E}$ & & \\
\hline Misobong (Litoral) & $1^{\circ} 29^{\prime} \mathrm{N}-9^{\circ} 46^{\prime} \mathrm{E}$ & (Centro-Sur) & \\
\hline
\end{tabular}


TABLE 1 (continued)

\begin{tabular}{|c|c|c|c|}
\hline $\begin{array}{l}\text { Parque Nacional de Monte Alén, } \\
\text { transect de Mosumo à } 500 \mathrm{~m} \\
\text { du début du layon (Centro-Sur) }\end{array}$ & $1^{\circ} 59^{\prime} \mathrm{N}-10^{\circ} 3^{\prime} \mathrm{E}$ & $\begin{array}{l}\text { Pico Surcado (Annobon) } \\
\text { Piedra Nzas, inselberg (Wele-Nzas) } \\
\text { Pradera de Baga (Litoral) }\end{array}$ & $\begin{array}{l}1^{\circ} 27^{\prime} \mathrm{N}-5^{\circ} 38^{\prime} \mathrm{E} \\
1^{\circ} 28^{\prime} \mathrm{N}-11^{\circ} 2^{\prime} \mathrm{E} \\
1^{\circ} 19^{\prime} \mathrm{N}-9^{\circ} 33^{\prime} \mathrm{E}\end{array}$ \\
\hline $\begin{array}{l}\text { Parque Nacional de Monte Alén, } \\
\text { transect de Mosumo à } 1420 \mathrm{~m} \\
\text { du début du layon (Centro-Sur) }\end{array}$ & $1^{\circ} 6$ 'N-10 $0^{\circ} 1^{\prime} \mathrm{E}$ & $\begin{array}{l}\text { Riaba (Bioko Sur) } \\
\text { Route forestière Engong-Jandje } \\
\quad \text { (Litoral) }\end{array}$ & $\begin{array}{l}3^{\circ} 23^{\prime} \mathrm{N}-8^{\circ} 46^{\prime} \mathrm{E} \\
1^{\circ} 25^{\prime} \mathrm{N}-9^{\circ} 32^{\prime} \mathrm{E}\end{array}$ \\
\hline $\begin{array}{l}\text { Parque Nacional de Monte Alén, } \\
\text { transect de Mosumo } \\
\text { (Centro-Sur) }\end{array}$ & $1^{\circ} 37^{\prime} \mathrm{N}-10^{\circ} 2^{\prime} \mathrm{E}$ & $\begin{array}{l}\text { Ruiché (Bioko Sur) } \\
\text { San Joaquin (Litoral) } \\
\text { Santa Isabel, pico Basile }\end{array}$ & $\begin{array}{r}3^{\circ} 24^{\prime} \mathrm{N}-8^{\circ} 33^{\prime} \mathrm{E} \\
2^{\circ} 2^{\prime} \mathrm{N}-9^{\circ} 56^{\prime} \mathrm{E} \\
3^{\circ} 35^{\prime} \mathrm{N}-8^{\circ} 46^{\prime} \mathrm{E}\end{array}$ \\
\hline $\begin{array}{l}\text { Parque Nacional de Monte Alén, } \\
\text { transect de Nkumékié, à } 4430 \text { m } \\
\text { du début du layon (Centro-Sur) }\end{array}$ & $1^{\circ} 29^{\prime} \mathrm{N}-10^{\circ} 18^{\prime} \mathrm{E}$ & $\begin{array}{l}\text { (Bioko Norte) } \\
\text { Sendjc (Litoral) } \\
\text { Sendje-Monte Mitra (Centro-Sur) }\end{array}$ & $\begin{array}{l}1^{\circ} 30^{\prime} \mathrm{N}-9^{\circ} 51^{\prime} \mathrm{E} \\
1^{\circ} 22^{\prime} \mathrm{N}-9^{\circ} 58^{\prime} \mathrm{E}\end{array}$ \\
\hline $\begin{array}{l}\text { Parque Nacional de Monte Alén, } \\
\text { transect de Nkumékié } \\
\text { (Centro-Sur) }\end{array}$ & $1^{\circ} 28^{\prime} \mathrm{N}-10^{\circ} 17^{\prime} \mathrm{E}$ & $\begin{array}{l}\text { Sendje-Ongamnsok (Litoral) } \\
\text { Ureca (Bioko Sur) } \\
\text { Utonde (Litoral) }\end{array}$ & $\begin{array}{l}1^{\circ} 22^{\prime} \mathrm{N}-9^{\circ} 58^{\prime} \mathrm{E} \\
3^{\circ} 15^{\prime} \mathrm{N}-8^{\circ} 35^{\prime} \mathrm{E} \\
1^{\circ} 56^{\prime} \mathrm{N}-9^{\circ} 49^{\prime} \mathrm{E}\end{array}$ \\
\hline
\end{tabular}

Previously recorded from Bioko (HUTCHINSON \& DALZIEL, 1954: 252; MORALES, 1994: 13; 1995: 17).

\section{2. *Amphiblemma molle Hook. f.}

CENTRO-SUR: Acurenam, Guinea \& Lope del Val 976 (MA 688882), 977 (MA 688881), 978 (MA 688880). Engong, dalle rocheuse, Parmentier \& Esono 2861, 2877 (BRLU). Evinayong, Guinea 250-509 (MA 688866). P.N. de Monte Alén, Lisowski 1437 (BRLU); ibidem, dalle rocheuse de Monte Alén, Nguema 465 (BRLU); ibidem, sur la piste lago Atoc-Moca, Senterre, Obiang \& Nguema 2153 (BATA, BRLU); ibidem, transect de Monte Chocolate, Van Reeth 140 (BRLU); ibidem, transect de Monte Alén. Lejoly 93/312, 94/123 (BRLU). WELE-NZAS: Mungum, inselberg, Parmentier \& Esono 3412 (BRLU). Obamico, Nguema \& Esono 292 (BATA, BRLU).

\section{Antherotoma irvingiana (Hook.f.) Jacq.-} Fél.

Centro-Sur: Bicurga, inselberg. Parmentier 1574bis, Parmentier \& Esono 3098 (BRLU). WELENzas: Akoak Ebanga, inselberg, Parmentier \& Esono 3491 (BRLU). Akuom, inselberg, Lisowski 663 (BRLU BATA), Obama \& Lejoly 628 (BATA, BRLU. MA612186). Asoc, inselberg, Obama \& Lejoly 480 (BATA, BRLU, MA-612111), Lejoly \& Esono 49. Parmentier \& Esono 3697 (BRLU). Dumu, inselberg Parmentier \& Esono 3622 (BRLU), Porembski, Biedienger \& Nguema 3599 (BRLU, BATA). Piedra Nzas, inselberg, Lejoly 99/202, 99/268 (BRLU).

Previously recorded from Río Muni (LejoLY, 1999b: 118, sub Dissotis irvingiana; PARMENTIER \& NGUEMA, 2001: 216 sub $D$. irvingiana; PARMenTIER \& al., 2001: 359; PARMENTIER, 2002: 915).
4. Calvoa crassinoda Hook. f. Calvoa molleri Gilg, C. uropetala Mildbr.

This species has been recorded from Annobón [Quioveo, Mildbraed 6528 (BATA), MIIDBRAED, 1922: 163 sub Calvoa uropetala; GUINEA, 1946: 341 sub C. uropetala; GUINEA, 1951a: 182, sub C. uropetala; HeDBERG \& HEDBERG, 1968: 134, BRENAN, 1979: 464 sub C. uropetala; MORALES, 1994: 15, sub C. uropetala; AEDO \& al. (1999) sub C. uropetala; Pic Santiago, à l'Ouest du lac de cratère, Descoings 12847 (P), 12848 (P), FiguEIREDO, 2001: 191)]. We could not examine any herbarium material of this species, but its distribution area suggest as probable its presence in Equatorial Guinea.

\section{Calvoa grandifolia Cogn.}

This species has been recorded from Annobón [Above Crater Lake on W side. Cliff sides in (not dense) wood. Wrigley \& Melville 164 (BM,K,MA,P), Figueireido 2001: 190]. We could not examine any herbarium material of this species, but its distribution area suggest as probable its presence in Equatorial Guinea.

\section{Calvoa hirsuta Hook. f.}

B Ioko SUR: Balachá-Ureca, Guinea 2297 (MA 268463), 2434 (MA 384327). Musola, Guinea 1189 (MA 384329). Moca, camino de Ureca, Fernández Casas 11775 (MA 555828); ibidem, Guinea 2079 (MA 
384328); ibidem, cascadas del Río lladyi, Carvalho 3266 (MA 555829). Belebú Balachá de Riaba, Fernández Casas 12141 (MA 555827). Centro-SuR: P. N. de Monte Alén, dalle rocheuse d'Engong, Lejoly 99/134, Parmentier \& Esono 2806, 2965, 2893 (BRLU); ibidem, dalle rocheuse de Monte Alén, Lejoly 99/048 (BATA, BRLU), Nguema 434, Parmentier \& Esono 3431, 3432 (BRLU); ibidem, transect de Monte Alén, Lejoly 94/117, 94/189, Van Reeth 43 (BRLU); ibidem, transect de Monte Chocolate, $2 \mathrm{~km}$ au NO Senterre \& Obiang 3822 (BRLU). Monte Mitra, Obama 350 (BATA, BRLU). LITORAL: Ncó-Misobong, grande cascade, Nguema \& Lejoly 185, 196 (BATA. BRLU). Sendje-Monte Mitra, Lejoly 01/22 (BRLU). WELE-NZAS: Obamico, Nguema, Esono \& Lejoly 279 (BATA).

Previously recorded from Bioko (HUTCHINSON, 1928: 209; GUINEA, 1946: 341; GuINEA, 1951a: 181; HutCHINSON \& DALZIEL, 1954: 251; MORALES, 1994: 13-15).

\section{7. *Calvoa jacques-felixii E. Figueiredo}

Вıко: Los Bimbiles, Guinea 244-140 (MA 689294).

\section{8. *Calvoa monticola A. Chev.}

CEnTRO-Sur: Bicurga, inselberg, Lisowski 1482, Parmentier 1613, (BRLU). Dumu, inselberg, Parmentier \& Esono 3600, 3797 (BRLU). P. N. de Monte Alén, dalle rocheuse d'Engong, Lejoly 99/155 (BATA, BRLU, MA621702), Lejoly 99/467bis, Parmentier \& Esono 2905, 2836, Stevart 1002, (BRLU); ibidem, transect de Monte Alén, Lejoly 94/188. Senterre \& Ngomo 2167 (BRLU); ibidem, transect de Mosumo, à $250 \mathrm{~m} 60^{\circ} \mathrm{NE}$ Senterre \& Ngomo 799 (BATA, BRLU). LITORAL: Bata - Sendje, km 40, Carvalho 5287 (BATA, MA-597976); Mbini, Lisowski 596 (BRLU). WELE-NZAS: Acasakira, rocher, Obama \& Lejoly 535 (BRLU); Akoak Ebanga, inselberg, Parmentier \& Esono 3518 (BRLU); Mungum, inselberg, Lejoly 99/036,99/367, 99/379, Lejoly \& Esono 75, Parmentier \& Esono 3340 (BRLU); Piedra Nzas, inselberg, Lejoly 99/196 (BATA, BRLU), Parmentier \& Nguema $375_{s t}$ (BRLU).

\section{Calvoa orientalis Taub.}

CEnTRO-SUR: Bicurga, inselberg, Lisowski 1387 , 1528 (BATA, BRLU).

Previously recorded from Rio Muni (GuINEA, 1946: 341).

\section{Calvoa pulcherrima Gilg ex Engl.}

CENTRO-SuR: Bicurga, inselberg, Parmentier 1561 (BRLU). P. N. de Monte Alén, dalle rocheuse d'Engong, Parmentier \& Esono 2982, Parmentier \& Nguema $294 \mathrm{st}$, 301 st, Stevart 995 (BRLU), Lejoly 99/100 (BATA. BRLU); ibidem, transect de Monte Alén, Lejoly 93/333 (BRLU), Wele-Nzas: Akoak Ebanga, inselberg, Parmentier \& Esono 3490 (BRLU). Akuom, inselberg,
Lisowski 676 (BATA, BRLU). Andom. inselberg, Lejoly 99/23I (BATA. BRLU), Lejoly 99/238 (BRLU). Asoc, inselberg, Parmentier \& Esono 3716 (BRLU). Bicurga, inselberg. Senterre \& Ngomo 483 (BRLU). Dumu, inselberg, Parmentier \& Esono 3596 (BRLU). Mfuin, inselberg, Obama \& Lejoly 367 (BATA, BRLU, MA 612042), Porembski, Biedinger \& Nguema 3503 (BATA, BRLU). Mungum, inselberg, Parmentier \& Esono 3402 (BRLU).

Previously recorded from Río Muni (LEJOLY \& LISOWSKI, 1999b: 116; PARMENTIER \& al., 2001: 351; PARMENTIER \& NGUEMA, 2001: 215; PARMENTIER, 2002: 915 ).

\section{1. *Calvoa sapinii De Wild.}

CENTRo-SuR: P. N. de Monte Alén, dalle rocheuse de Monte Alén, Parmentier \& Esono 2964 (BRLU).

12. *Calvoa seretii Jacq.-Fél. subsp. seretii LITORAL: Etembue, Lisowski 554 (BATA, BRLU).

\section{Calvoa trochainii Jacq.-Fél.}

This species has been recorded from Bioko Sur [Akpabla, GC 11492 (K), Musola, Guinea 930bis (K, MA 384308), 935bis (MA 384307), FIGUEIREIDO, 2001: 186]. We could not examine any herbarium material of this species, but its distribution area suggest as probable its presence in Equatorial Guinea.

\section{Dicellandra barteri Hook.f.}

D. escherichii Gilg ex Engl., D. scandens Gilg ex Engl.

Different varieties of Dicellandra barteri Hook. f. were distinguished by JACQUESFÉLIX (1974b). We do not recognize varieties in this work and moreover, lots of specimens do not correspond to any of them:

Bioko Norte: Monte Balea, en la carretera de Malabo (Santa Isabel) a Laka, Guinea 520 (MA 384326). Broko Sur: Ureca, Guinea 2319 (MA 384322), 2323 (MA 384319). Centro-Sur: Evinayong, Guinea 239-507 (MA 688872). Monte Alén, transect Ecofac de Mosumo, à $250 \mathrm{~m} 60^{\circ} \mathrm{NE}$ du transect, Senterre \& Ngomo $267 \mathrm{st}$ (BATA, BRLU); ibidem, transect de Ncumekie, Lejoly 95/176 st. Litoral: Mayang, Lisowski 787st, 881 (BRLU). Route forestière Engong-Jandje, Lejoly \& Van Asbroeck 11 si (BATA, BRLU).

The following specimens could be attributed to the var. barteri:

Bioko Norte: Malabo a Cupapa, km 22-23, Carvalho 3405 (MA 558404), 3411 (MA 555830). Carretera del 
Pico Basilé, Carvalho 4465 (MA 555831). Bıoko SUR: Ureca. Guinea 2321. CENTRO-SuR: Monte Alén, Lisowski 1547 (BRLU). LiTORAL: Bata-Niefang, km 35, Carvalho 5960 (BATA, MA 688870). Ncoho, Nguema 135 (BRLU). Wele-Nzas: Nsum Esangüi, Pérez Viso 3647 (MA 688874). Montée inselberg Akuom, Lisowski 697 (BATA, BRLU), Obama \& Lejoly 409 (BRLU, MA 612116).

The following specimens could be attributed to the var. escherichii:

Centro-Sur: Monte Alén, Ngomo 241 (BATA, BRLU). P. N. de Monte Alén. dalle rocheuse d'Engong, Parmentier \& Esono 2875 (BRLU); ibidem, cabaña del lago,Velayos, Aedo \& Pérez-Viso 9524 (MA 688870); ibidem, transect de Nkumékié à $2550 \mathrm{~m}$ du layon, Senterre \& Ngomo 16 (BRLU). Sendje-Monte Mitra, Lejoly 01/20 (BRLU). LITORAL: Mbambala. río, Nguema \& Lejoly 9 (BATA, BRLU). WeLE-Nzas: Ngong Mocomo, Lejoly \& Elad 98/036 $6_{s t}$ (BRLU).

Previously recorded from Bioko (HuTCHINSON, 1928: 210; GUINEA, 1946: 341, 344; GUINEA, 1951a: 184; HUTCHINSON \& DALZIEL, 1954: 252; HEDBERG \& HEDBERG, 1968: 130; Morales, 1994: 15; MoRales, 1995: 17) and Río Muni (GUINEA, 1946: 341 sub Dicellandra escherichii).

\section{5. *Dicellandra descoingsii Jacq.-Fél.}

Bloko Norte: Malabo-Laka, Guinea 361 (MA 268686). Centro-Sur: À quelques kilomètres de l'inselberg de Bicurga, Parmentier \& Esono 3316 (BRLU). P. N. de Monte Alén, chemin vers la dalle rocheuse de Engong, Parmentier \& Esono 2916 (BRLU); ibidem, dalle rocheuse d' Engong, Parmentier \& Esono 2867 (BRLU); ibidem, dalle rocheuse de Monte Alén, Parmentier \& Esono 3448 (BRLU). LITORAL: Mandjani, Nguema 86 (BRLU). Mayang, Lisowski 797 (BRLU). WELE-NZAS: Obamico (Parque N. de Nsork), Nguema, Esono \& Lejoly 278 (BATA, BRLU).

\section{Dichaetanthera africana (Hook. f.) Jacq.-Fél. Sakersia africana Hook. f.}

Bioko-Sur: Belebú Balachá, Fernández Casas 12114 (MA 555980). Belebú Balachà -Ureca, km 3, Carvalho 2523 (BRLU, MA-555852). BIOKO-NORTE: Baríobe, Fernández Casas 11S16 (MA 555855). Carretera del pico Basilé, Fernández Casas 11236 (BRLU, MA 555854). Malabo-Riaba, km 14-15, Carvalho 3868 (BRLU, MA-555853). CENTRO-SUR: Bicurga, inselberg, Lisowski 1385st (BATA, BRL.U). Dumu, inselberg, Parmentier \& Esono 3625. Evinayong Cogo, Guinea 493II8 (MA 688892). Monte Alén, Lisowski 1432 st (BRLU). P. N. de Monte Alén, transect de Monte Alén, Lejoly 93/350 st (BRLU). LITORAL: Bata-San Joaquin, $4 \mathrm{~km}$ de San Joaquin, Carvalho 4969 (BRLU, MA
599206). Sendje-Ongamnsok, Lejoly 01/04 (BRLU). Wele-Nzas: Aconibe, Lejoly 99/276 (BATA, BRLU). Dumu, inselberg, Lejoly 99/350 st (BATA, BRLU). Mungum, inselberg, Lejoly 99/373T1 (BRLU).

Previously recorded from Bioko (Fernández CASAS \& MORALES, 1995: 237; MORALES, 1994: 15) and from Río Muni (Hutchinson, 1928: 209 sub Sakersia africana; GUINEA, 1946: 341 sub S. africana; HUTCHINSON \& DALZIEL, 1954: 249 sub $S$. africana).

\section{Dinophora spenneroides Benth.}

Centro Sur: Bicurga, inselberg, Parmentier 1695. Parmentier \& Esona 3151 (BRLU). Mosumu, Obama \& Lejoly 683 (BRLU), Pérez Viso 3483 (MA 688883); ibidem, cataratas del río Uoro, Pérez Viso 376 (MA 688878): ibidem, confluence Río Laña avec Río Mbini, Obama \& Lejoly 683 (BRLU). Oyac Esom, Pérez Viso 3450 (MA 688885). P. N. de Monte Alén, Ngomo 75 (BATA, BRLU); ibidem, transect de Nkumékié à $4225 \mathrm{~m}$ du début du layon, Senterre \& Ngomo 38 (BATA, BRLU). KIE-NTEM: Mfuin, inselberg, Obama \& Lejoly 358 (BATA, BRLU). LITORAL: Aeródromo, Guinea 463 (MA 688893). Ayamiken, Lejoly 97/088, Obama 180 (BRLU). Bata-Río Campo, km 48-49, Carvalho 4675 (BATA, MA-597455). Bata-Niefang, Carvalho 5574 (MA 598565). Bata-Sendje, km 23-24, Carvalho 5255 (BATA, MA 598014). Ecucu, Guinea 252 (MA 688896). Pradera de Baga, Eneme 362 (BATA, BRLU). Utonde, Guinea 40I (MA 688895), 403 (MA 688894). WELENzAS: Aconibe, Obama 306 (BATA, BRLU). Akoak Ebanga, inselberg, Parmentier \& Esono 3581 (BRLU). Akuom, inselberg, Lisowski 698 (BRLU). Explotación forestal de Añisok Mongola, Pérez Viso 3756 (MA 688877). Asoc, inselberg, Lejoly 99/314, Parmentier \& Esono 3674 (BRLU). Ayene, Lisowski 742 (BATA, BRLU). Dumu, inselberg, Parmentier \& Esono 3827 (BRLU). Kukumankok, entre le village et l'inselberg Mongum, Lejoly \& Esono 69 (BRLU). Mungum, inselberg, Parmentier \& Esono 3388 (BRLU). Ngong Mocomo, vers inselberg Acoak Banga, Lejoly \& Elad 981007 (BATA, BRLU). Entre Nsuameyong et inselberg Akuom, Obama \& Lejoly 455 (BATA, BRLU).

Previously recorded from Equatorial Guinea (without locality) (GUINEA, 1951a: 184), Bioko (GUINEA, 1946: 340-341; HUTCHINSON, 1928: 205; HutChINSON \& DALZIEL, 1954: 252) and Río Muni (GUINEA, 1946: 178, 340-341; LEJOLY \& LISOWSKI, 1999b: 119).

\section{8. *Dissotis barteri Hook. f.}

WELE-NZAS: Akoak Ebanga, inselberg, Lejoly \& Elad 98/077 (BATA, BRLU); Parmentier \& Esono 3495, 3544 (BRLU). 
19. Dissotis congolensis (Cogn. ex Büttner) Jacq.-Fél.

Osbeckia congolensis Cogn. ex Büttner

LITORAL: Bata-Bome. Carvalho 4670 (BATA, MA 597518). Au sud de Bata, au sud du fleuve Ekuka, Lisowski 1580. Etembue, Lejoly \& Elad 98/202 (BRLU).

Previously recorded from Río Muni (GUINEA, 1946: 178 sub Osbeckia congolensis; LEJOLY \& LISOWSKI, 1999c: 185).

\section{Dissotis hensii Cogn.}

CEnTro-Sur: Akoak Ebanga, inselberg, Parmentier \& Esono 3578. Bicurga, inselberg, Lisowski 1374 (BATA, BRLU). Monte Alén, Lisowski 1450 (BRLU), Ngomo 356 (BATA, BRLU). WELE-NZAS: Dumu, inselberg, Parmentier \& Esono 3829 (BRLU). Mfuin, inselberg, Obama \& Lejoly 355 (BATA, BRLU), 356 (BRLU, MA 612043). Entre Nsuameyong et inselberg Akuom, Obama \& Lejoly 449 (BRLU, MA-612034). Route de Ngong Mocomo vers Ovong, Lejoly \& Elad 98/086 (BATA, BRLU).

Previously recorded from Río Muni (PARMENTIER, 2002: 915).

\section{Dissotis multiflora (Sm.) Triana}

Bıoko: Malabo-Riaba, km 26, Carvalho 2976 (MA 555834). CENTRO-SUR: Acurenam, Guinea \& Lope del Val 979 (MA 688879). Bicurga, inselberg, Lisowski 1374bis, Parmentier \& Esono 3152 (BRLU). Engong, dalle rocheuse, Parmentier \& Esono 2906 (BRLU). Evinayong, Guinea 483-122 (MA 688868), 482-123 (MA 688869). P. N. de Monte Alén, transect de Monte Chocolate, Lejoly 95/139 (BRLU). Monte Alén, Ngomo 321 (BATA, BRLU). KIE-NTEM: Mfuin, inselberg, Obama \& Lejoly 356 (BRLU). LTTORAL: Ayamiken, Lejoly 97/013, Lisowski 948 (BATA, BRLU). Elende, Lisowski 1042 (BATA, BRLU). Ngoma, Lejoly \& Elad 98/124 (BRLU). Sendje, Pérez Viso 3369 (MA 688887). SendjeOngamnsok, Lejoly 01/86 (BRLU). WELE-NZAS: Entre Nsuameyong et inselberg Akuom, Obama \& Lejoly 449 (BATA, BRLU). Akuom, inselberg, Lisowski 729 (BATA, BRLU). Ngong Mocomo, vers inselberg Acoak Ebanga, Lejoly \& Elad 98/006 (BRLU).

Previously recorded from Bioko (MORALES, 1994: 15; FERnÁNDEZ CASAS \& MoRALES, 1995: 237).

\section{*22. Dissotis thollonii Cogn.}

Centro-Sur: P. N. de Monte Alén, dalle rocheuse, Lejoly 99/004, 99/435 (BATA, BRLU), Parmentier 1530 st, Parmentier \& Esono 2721, 2763, 3453 (BRLU).

\section{Guyonia ciliata Hook. f.}

Bioko SuR: Desembocadura del lago de Biac, Fernández Casas 11972 (BRLU, MA 552726). CENTRO-
SuR: P. N. de Monte Alén, dalle rocheuse d'Engong, Lejoly 99/083, 99/390 (BATA, BRLU); ibidem, dalle rocheuse de Monte Alén, Lejoly 99/023 (BATA, BRLU), Parmentier 1512 (BRLU).

Previously recorded from Bioko (HUTCHINSON \& DALZIEL, 1954: 246; MORALES, 1994: 17).

24. Heterotis arenaria Jacq.-Fél.

Heterotis obamae Lejoly \& Lisowski [comparison of the types indicate that this species is a synonym for the previously described Heterotis arenaria Jacq.-Fél.]

LITORAL: Etembue, Guinea 227-915 (MA 688867), Lejoly \& Elad 98/112 (BATA, BRLU), Lisowski 527 (BRLU). Pradera de Baga, Eneme \& Lejoly 132 (BATA, BRLU).

Previously recorded from Río Muni (LejoLY \& LISOWSKI, 1999: 185 sub Heterotis obamae).

25. *Heterotis buettneriana (Cogn.) Jacq.Fél.

LITORAL: Bata-Sendje, km 38, Carvalho 6279 (MA 620439).

26. Heterotis decumbens (P. Beauv.) Jacq.Fél.

Dissotis decumbens (P. Beauv.) Triana

Bioko Norte: Bahfa de Venus, Guinea 256 (MA 384323), 322 (MA 268690), 330 (MA 384324). B1OKO SUR: Belebú Balachá de Riaba, Fernández Casas 12122 (MA 555835). Playa de Ureca, Guinea 2464 (MA 384325). Centro Sur: Enkumekie, Pérez Viso 3507 (MA 688875). Monte Alén, Ngomo 94 (BATA, BRLU). LITORAL: Bata-Bome, Carvalho 4668 (BATA, BRLU, MA 597516). Bata, Nguema 1064 (BRLU); ibidem, près du fleuve Ekuka, Lisowski 1219 (BATA, BRLU); ibidem, Costa Ubonde, Guinea \& Lope del Val 229 (MA 688873). Corisco, isla, Obama 733 (BRLU). Etembue, Eneme \& Lejoly 68, Lejoly \& Elad 98/III (BATA, BRLU). Jandje, Lisowski 302 (BRLU). Mboete. Eneme 374 (BATA, BRLU). Pradera de Baga, Eneme \& Lejoly 93 (BATA, BRLU). WELE-NzAs: Mungum, inselberg, Lejoly 99/365, Parmentier \& Esono 3378, 3582 (BRLU). Route de Ngong Mocomo vers Ovong, Lejoly \& Elad 981091 (BATA, BRLU).

Previously recorded from Bioko (GUINEA, 1946: 176, 340 sub Dissotis decumbens; GUINEA, 1951a: 180-181 sub $D$. decumbens; HUTCHINSON \& DALZIEL, 1954: 257 sub $D$. decumbens; MORALES, 1994: 15-17 sub $D$. de- 
cumbens) and Río Muni (LEJOLY \& LISOWSKI, 1999c: 185).

\section{Heterotis prostrata Benth.}

BIOKO NORTE: Malabo-Basilé, km 11, Carvalho 2091 (MA 555836): Malabo-Riaba, Fernández Casas 10230 (MA 555837), Carvalho 2008 (MA 558402). Bioko SUR: Moca, Lope de Val s.n. (MA 500350). Moca-lago Loreto, Fernández Casas 11876 (MA 555832). Moca-Riaba, km 1, Fernández Casas 11567 (MA 558403). Moca-Ureca, Fernández Casas 11748 (MA 555833). Musola, Guinea 1295 (MA 384346), 1347 (MA 384347), 1387 (MA 384336), 1396 (MA 384337); ibidem, camino de Monkey Bush, 981 (MA 268691), 1070 (MA 384345). CENTRO-SUR: Bicurga, inselberg, Lejoly 99/492 (BATA, BRLU), Parmentier \& Esono 3097, 3150 (BRLU). Monte Alén, dalle rocheuse, Lejoly 99/028 (BATA, BRLU, MA 621780). P. N. de Monte Alén, Engong, dalle rocheuse, Lejoly 99/082, 99/102 (BATA, BRLU), Parmentier \& Esono 2727 (BRLU). WeLE-NZAS: Mungum, inselberg, Lejoly \& Esono 76 (BRLU).

Previously recorded from Río Muni (LejoLY \& LISOWSKI, 1999c: 188; PARMENTIER, 2002: 915).

The specimens of $H$. rotundifolia $(\mathrm{Sm}$. Jacq.-Fél. and Dissotis fruticosa (Brenan) Keay we examined do not show sufficent differences with $H$. prostrata. We decided to include them under $H$. prostrata. As we have not compared the type material of the three species, we do not put them in synonymy. Nevertheless we consider the species $D$. fruticosa as uncertain as well as the presence of $H$. rotundifolia in Equatorial Guinea.

\section{Heterotis rupicola (Gilg ex Engl.) Jacq.- Fél.}

Centro-Sur: P. N. de Monte Alén, dalle rocheuse d'Engong, Lejoly 99/081 (BATA, BRLU, MA-621788), Parmentier \& Esono 2983 (BRLU); ibidem, dalle rocheuse de Monte Alén, Lejoly 99/032 (BATA, BRLU), Parmentier 1510 (BRLU).

Previously recorded from Río Muni (LejoLY \& LISOWSKI, 1999c: 188; PARMENTIER \& Nguema, 2001: 216; PARMENTIER \& al., 2001: 350; PARMENTIER, 2002: 914).

29. *Lijndenia jasminoides (Gilg) Borhidi

Annobon: Aual-Palé, $2,5 \mathrm{~km}$ de Aual, Carvalho 3110 (MA 558407).

\section{Medillina mannii Hook. f.}

Bioko SuR: Ruiché-Gran Caldera de Luba, Carvalho 3834 (MA 555848). CENTRO-SuR: P. N. de Monte Alén, dalle rocheuse d'Engong, Parmentier \& Esono 2798, Stevart 1010 (BRLU). WELE-NzAS: Mungum, inselberg, Parmentier \& Esono 3394bisst (BRLU).

Previously recorded from: Bioko (HUTCHINSON, 1928: 209; GUINEA, 1946: 341; HuTCHINSON \& DALZIEL, 1954: 251; JoHANSSON, 1974: 42; MORALES, 1994: 17) and Río Muni (GUINEA, 1946: 157).

\section{Medillina mirabilis (Gilg) Jacq.-Fél. Myrianthemum mirabile Gilg}

Censtro-Sur: P. N. de Monte Alén, transect de Nkumékié, Lejoly 95/174 (BRLU); ibidem, près de la Cabaña de Esamalan, Senterre, Obiang \& Ngomo 2123 (BRLU). LITORAL: Etembue, Lejoly \& Van Asbroeck 35 (BRLU).

Previously recorded from Bioko (GUINEA, 1946: 341 sub Myrianthemum mirabile; HuTCHINSON \& DALZIEL, 1954: 247 sub M. mirabile) and Río Muni (GunNEA, 1946: 341 sub M. mirabile).

\section{2. *Memecylon aequidianum Jacq.-Fél.}

LITORAL: Etembue, Eneme \& Lejoly 42 (BATA, BRLU). Wele-NzAS: Piedra Nzas, inselberg, Nguema \& Parmentier 509 (BRLU).

\section{3. *Memecylon candidum Gilg}

Centro-Sur: Bicurga, inselberg, Stevart 913 (BRLU).

\section{Memecylon collinum Jacq.-Fél.}

KIE-NTEM: Mfuin, inselberg, Obama \& Lejoly 375st (BATA, BRLU). WELE-NZAS: Akoak Ebanga, inselberg, Parmentier \& Esono 3500, 3519 (BRLU). Dumu, inselberg, Lejoly 99/331 st, 99/349T1 st (BRLU), Parmentier \& Esono 3796 (BRLU). Mungum, inselberg, Lejoly 99/368 (BATA, BRLU, MA 621697), Lejoly \& Esono 86 (BRLU). Piedra Nzas, inselberg, Lejoly 99/185 st (BRLU).

Previously recorded from Río Muni (LEJOLY \& LISOWSKI, 1999b.: 120; PARMENTIER \& NGUema, 2001: 210; PARMentier \& al., 2001: 352; PARMENTIER, 2002: 915).

\section{5. *Memecylon klaineanum Jacq.-Fél.}

Centro-Sur: P. N. de Monte Alén, transect de Mosumo, à $1420 \mathrm{~m}$ du début du layon, Senterre \& Ngomo 801, 805 (BRLU). 


\section{6. *Memecylon laurentii De Wild.}

Centro-Sur: P. N. de Monte Alén, transect de Monte Alén, Senterre, Obiang \& Esono 2817 (BRLU); ibidem, près du village de Monte Alén (Ecofac), colline entourée par le sentier pédagogique, Senterre, Obiang \& Esono 2848. Monte Mitra, Obama 346 (BATA, BRLU). LITORAL: Bata-Bome, Carvalho 5299 (BATA, BRLU, MA 598114).

37. *Memecylon nodosum (Engl.) Gilg ex Engl.

Centro-Sur: P. N. de Monte Alén, près de la Cabaña Ecofac de Misergue, Senterre \& Obiang 3305 (BRLU).

\section{8. *Memecylon salicifolium Jacq-Fél.}

LITORAL: Bata-Sendje, km 62-63, Carvalho 644 I (BATA, BRLU, MA 635355).

\section{9. *Memecylon virescens Hook. $f$.}

CENTRo-Sur: Bata-Niefang, km 35, Carvalho 5684 (BRLU. MA 598987). Monte Alén. Ngomo 423 (BATA, BRLU). LITORAL: Handje, Lejoly \& Elad 98/184 (BATA, BRLU).

40. *Memecylon viride Hutch. \& Dalz.

Centro-Sur: P. N. de Monte Alén, transect de Mosumo, Van Reeth 334 (BRLU).

\section{1. *Memecylon zenkeri Gilg}

Centro-Sur: P. N. de Monte Alén, transect de Monte Chocolate, Lejoly 95/146, Senterre \& Ngomo 3419 (BRLU).

\section{Ochtocharis dicellandroides (Gilg) C. Hansen \& Wickens \\ Phaeoneuron dicellandroides Gilg}

LTTORAL: Bata-Mbini, km 24, Carvalho 6367 (MA 620603). Bata-Niefang, km 38. Carvalho 5407 (BATA, BRLU, MA 598315). Midjomitom, Carvalho 6325 (BATA, BRLU, MA 620562). WELE-NzAS: Dumu, inselberg, Nguema 187 (BRLU). Mungum, inselberg, Parmentier \& Esono 3410 (BRLU).

Previously recorded from Río Muni (GuINEA, 1946: 341 sub Phaeoneuron dicellandroides).

\section{Preussiella kamerunensis Gilg Preussiella chevalieri Jacq.-Fél.}

Bioko Norte: Carretera del Pico Basilé, km 4, Carvalho 3527 (MA 555849), 4506 (MA 555851). BıKo Sur: Belebú Balachá de Riaba a Ureca, km 4, Carvalho 4203 (MA 555850).
Previously recorded from: Bioko (MoRALES, 1994: 17; FERnÁndeZ CASAS \& MoRALES, 1995: 237).

\section{4. *Spathandra blakeoides (G. Don) Jacq.-Fél.}

LITORAL: Etembue, Eneme \& Lejoly 37, Lisowski 514 (BATA, BRLU). Au $S$ de Bata, près du fleuve Ecucu, Lisowski 1209 (BRLU). Jandje, Lisowski 35, 284 (BRLU). Kogo, Obama 889 (BATA, BRLU). Mayang, Lisowski 813 (BATA, BRLU).

\section{Tristemma demeusei De Wild.}

Tristemma radicans Gilg ex Engl.

CenTro-Sur: Engong, dalle rocheuse, Lejoly 99/088, Parmentier \& Esono 2739, 2937st, $2972_{\text {st }}$ (BRLU). P. N. de Monte Alén, dalle rocheuse, Lejoly 99/019, Parmentier $1515_{s t,} 3039 s t$ (BRLU), Lejoly 99/391 (BATA, BRLU); ibidem, transect de Monte Alén, Van Reeth 72 (BRLU); ibidem, transect de Nkumékié à $2550 \mathrm{~m}$ du début du layon. Senterre \& Ngomo 37. (BRLU). WeLENzAS: Ayene, Lisowski 757 (BRLU).

Previously recorded from: Bioko (GuINEA, 1946 sub Tristemma radicans: 340; HUTCHINSON \& DALZIEL, 1954: 250 sub T. radicans).

\section{Tristemma hirtum P. Beauv.}

Bioko SuR: Moca, Lope de Val s.n. (MA 500350); ibidem, hacia las cascadas de Iladyi, Carvalho 4093 (MA 555810). Moca-lago Biaó, Fernández Casas 11916 (MA 555813). LTTORAL: Abdelmang, Guinea \& Lope del Val 663 (MA 688888). Bata, Nguema 1167. Bolondo, Guinea 256-689 (MA 688901). Costa Bata. Guinea \& Lope del Val 661 (MA 688889). Ecucu, Guinea 259-75I (MA 688898), 409 (688900). Elendé, Lisowski 1043 (BRLL). Eyan Bot, km. 12 de la carretera del puerto a Cogo, Pérez Viso 3541 (MA 688876). Etembue, Guinea 423 (MA 688899), Lejoly \& Elad 98/14, 98/201 (BATA, BRLU), Lisowski 508 (BATA, BRLU), 549 (BRLU). Jandje, Lisowski 41 (BATA, BRLU). Pradera de Baga, Eneme 361, Eneme \& Lejoly 141 (BRLU). WELE-NzAS: Asoc, inselberg, Obama \& Lejoly 475 (BATA, BRLU).

Previously recorded from: Bioko (HuTCHINSON, 1928: 208; GUINEA, 1946: 340; GUINEA, 1951a: 176; HUTCHINSON \& DALZIEL, 1954: 250; MORALES, 1994: 17-18; MORALES, 1995: 18) and Río Muni (GuINEA, 1946: 340; LEJOLY \& LISOWSKI, 1999b: 120; LEJOLY \& LISOWSKI, 1999c: 185; PARMENTIER \& NGUEMA, 2001: 216).

47. Tristemma littorale subsp. biafranum Jacq.-Fél.

BIOKo NORTE: Boopa, entre Malabo y Pico Basilé, 
Castroviejo 9095SC (MA 688890). Carretera del pico Basilé, km 5, Carvalho 3621 (MA 555818); ibidem, km 22, Carvalho 2210 (MA 555817). BIoko SUR: Luba, Fernández Casas 12035bis (MA 555823). Desde la carretera general a la playa de Arenas Blancas, Fernández Casas 11647 (MA 555821). Musola, Guinea 1263 (MA 384369), 1265 (MA 270517), 1319 (MA 384374), 1320 (MA 384375). Cerca del cruce de Moca, Fernández Casas 11442 (MA 555820). LITORAL: Ayamiken, Lejoly 97/036 (BATA, BRLU), Lisowski 432 (BRLU). Etembue, Lisowski 501 (BRLU), 562, 1132 (BATA, BRLU). Mbambala, río, Nguema \& Lejoly 5 (BATA, BRLU). Mboete, Eneme 378 (BATA, BRLU). Jandje, Lisowski 305, 1322 (BRLU). Sendje, Lisowski I75 (BRLU). WELE-NZAS: Aconibe, Obama 305 (BATA, BRLU).

Previously recorded from Río Muni (LEJOLY \& LISOWSKI, 1999a: 106; LEJOLY \& LISOWSKI, 1999c: 185).

\section{Tristemma littorale Benth. subsp. litto- rale}

AnNobon: Pico Surcado, Wrigley 65 (MA 270518). Bıoko SuR: Luba-Veiga y Avendaño, Fernández Casas 12045 (MA 555822). Malabo-Luba, Carvalho 2050 (MA 555815). Ureca, Guinea 2514 bis (MA 384370). ВIOKO NORTE: Malabo, puerto marítimo, Carvalho 4114 (MA 555816)

Previously recorded from: Bioko (HuTCHINSON, 1928: 208; GUINEA, 1951a: 178; HuTCHINSON \& DALZIEL, 1954: 250; HEDBERG \& HEDBERG, 1968: 130; MORALES, 1994: 18; MORALES, 1995: 18) and Río Muni (GuINEA, 1946: 176b; 340).

\section{Tristemma mauritianum J.F. Gmel. T. incompletum $\mathrm{R}$.Br..}

BIoko Sur: Moca-lago Biaó, Fernández Casas 10373 (MA 555812), 11711 (MA 555811). Moca-Riaba, por el camino viejo, Fermández Casas 11845 (MA 555814). Carretera de Moca, Guinea 1902 (MA 270516). Musola, lago Loreto, Guinea 1839 (MA 384367). CENTRO-SUR: Bicaba, Pérez Viso 3459 (MA 688884). P. N. de Monte Alén, dalle rocheuse d'Engong, Lejoly 99/066 (BATA, BRLU, MA 621708), Lejoly 99/451.2, Parmentier \& Esono 2974 st (BRLU); ibidem, Esamalang. Pérez Viso 3308 (MA 688886). LITORAL: Ayamiken, Lejoly 97/035, Lisowski 911, Obama 159. (BATA, BRLU). WELENzas: Akoak Ebanga, inselberg, Parmentier \& Esono 3579 (BRLU), Asoc, inselberg, Lejoly 99/310 (BATA, BRLU). Dumu, inselberg, Lejoly 99/364, Parmentier \& Esono 3640, 3828 (BRLU). Obamico (Parque N. de Nsork), Nguema \& Esono 286 (BATA, BRLU).

Previously recorded from Bioko (GuINEA, 1951: 176-178 sub Tristemma incompletum; MORALES, 1994: 18; 1995: 18).

\section{0. *Tristemma oreophilum Gilg}

Bioko Norte: Cupapa, Fernández Casas 11389. Bioko SuR: Malabo-Luba-Moca, estrada km 68, Carvalho 2615 (MA 400268). CENTRO-SUR: P. N. de Monte Alén, Lejoly 99/156 (BRLU); ibidem, chemin vers dalle rocheuse d' Engong. Parmentier \& Esono 2907 (BRLU); ibidem, dalle rocheuse de Monte Alén, Lejoly 99/389 (BATA, BRLU); ibidem, $2 \mathrm{~km}$ au NE du site de traversée du Río pour aller aux cataractas, Senterre, Obiang \& Ngomo 2068 (BRLU); ibidem, entre la station Ecofac de Mosumo et Monte Boracho, Senterre \& Nogmo 290 (BRLU). LITORAL: Mboete, Eneme 386 (BRLU). Ncó, Nguema \& Lejoly 156 (BRLU, MA-611887). WELENZAS, Ngong Mocomo, Parmentier \& Esono 3580 (BRLU).

\section{1. *Tristemma rubens A. \& R. Fern.}

Centro-Sur: Bicurga, inselberg, Lisowski 1487 (BRLU). LITORAL: Bata-Senge, km 27, Carvalho 6167 (MA 619175). Nco-Misobong au niveau de la grande cascade, Nguema \& Lejoly 198 (BATA, BRLU, MA 611882).

\section{2. *Warneckea bebaiensis (Gilg ex Engl.) Jacq.-Fél.}

Centro Sur: P. N. de Monte Alén, transect de Nkumékié, à $4430 \mathrm{~m}$ du début du layon. Senterre \& Obiang 3649 (BRLU).

\section{3. *Warneckea cauliflora Jacq.-Fél.}

Centro Sur: Parc National de Monte Alén, transect Ecofac de Nkumékié, à $5300 \mathrm{~m}$ du début du layon, Senterre \& Obiang 3604 st (BRLU).

\section{4. *Warneckea fosteri (Hutch. \& Dalz.) Jacq.-Fél.}

AnNOBON: Ridge W of Crater Lake, Wrigley 201 (MA 268763). Palé, Canvalho 3144 (MA 558408). LitoraL: Bata-Mbini, km 27, Carvalho 6086 (MA 618989).

\section{Warneckea membranifolia (Hook. f.) Jacq.-Fél. \\ Memecylon membranifolium Hook. f.}

Centro Sur: P. N. de Monte Alén, transect de Nkumékié à $2650 \mathrm{~m}$ du début du layon, Senterre \& Ngomo 15 (BRLU); ibidem, transect de Nkumékié à $3200 \mathrm{~m}$ du début du layon, Senterre \& Ngomo 25 (BRLU). WELENZAS: Akoak Ebanga, inselberg, Parmentier \& Esono 3560 (BRLU). Kukumankok, entre l'inselberg Mungum et le village, Lejoly \& Esono 57.

Previously recorded from Bioko (HuTCHINSON, 1928: 215 sub Memecylon membranifolium; GUINEA, 1946: 341 sub $M$. membranifolium; HUTCHINSON \& DALZIEL, 1954: 
263 sub $M$. membranifolium) and Río Muni (PARMENTIER \& al., 2001: 362).

\section{Warneckea memecyloides (Benth.) Jacq.-Fél. \\ Memecylon memecyloides (Benth.) Exell}

Bioko Norte: Ela Nguema, barrio de Santa Cruz, Carvalho 3398 (MA 688891), 3423 (MA 558406).

Previously recorded from Annobón (FERNÁNDEZ CASAS \& MORALES, 1995: 237 sub Memecylon memecyloides) and Bioko (HuTCHINSON \& DALZIEL, 1954: 263; MoRALES, 1994: 17; 1995: 17).

\section{7. *Warneckea pulcherrima (Gilg) Jacq.- Fél.}

Centro Sur: Bicurga, inselberg, Parmentier \& Esono 3214 (BRLU). P. N. de Monte Alén, $2 \mathrm{~km}$ au NE du site de traversée du Río pour aller aux cataractas, Senterre, Obiang \& Ngomo 2364 (BRLU); ibidem, transect de Monte Chocolate, Lejoly 95T/L3.1023 (BRLU).

\section{Doubtful records}

No specimen supporting the following records has been found:

Memecylon englerianum Cogn. recorded in Río Muni (GUINEA, 1946: 341). This species is distributed over Nigeria and Western Cameroon.

Memecylon erythranthum Gilg recorded in Río Muni (GUINEA, 1946: 146). This species is distributed in Tanzania and Zimbabwe; its presence in Equatorial Guinea is not very probable.

Osbeckia tubulosa $\mathrm{Sm}$. recorded in Río Muni (GUINEA, 1946: 184). This species is distributed from Guinea to Nigeria; its presence in Equatorial Guinea is not very probable.

Tristemma albiflorum Benth. recorded in Río Muni (GUINEA, 1946: 184). This species is distributed from Senegal to Ghana, its presence in Equatorial Guinea is not very probable.

We prefer not to consider those species in the check list as long as no material is collected in Equatorial Guinea.

\section{Insufficently known species}

Memecylon fernandianum Gilg ex Engl. has been recorded from Bioko (GuINEA, 1946: 341; HuTCHINSON \& DALZIEL, 1954: 263).

Memecylon tessmannii Gilg ex Engl. recorded in Río Muni (GuinEA, 1946: 341).
Memecylon vogelii Naudin recorded in Bioko (HuTCHINSON, 1928: 215; GUINEA, 1946: 341).

Tristemma oreosthamnos Mildbr. has been recorded from Annobón (EXCELL, 1963: 100; GUINEA, 1946: 340; 1951: 178; HEDBERG \& HEDBERG, 1968: 134; MORALES, 1994: 18).

We could not examine any specimens of those species. As they are insufficently known (LEBRUN \& STORK, 1991) we prefer not to consider them in the check list.

\section{Repartition of species between Bioko,} Annobón and Río Muni

The species repartition is given in table 2 , as well as the indications for the $\mathbf{2 6}$ new species for the country, for Río Muni (29), Bioko (6) and Annobón (3). Fifty-seven species were found in Equatorial Guinea, 49 in Río Muni, 23 in Bioko and 6 in Annobón. The high number of species newly recolted in the country and the fact that several species are represented by one or few herbarium specimen, indicates that the prospection should be continued.

\section{Collection index}

The species is indicated by a number in parenthesis corresponding to the number in the check list.

Carvalho: 2008 (27); 2050 (48); 2091 (27); 2210 (47); 2484 (1); 2523 (16); 2615 (50); 2617 (1); $2976(21) ; 3110$ (29); 3144 (54); 3266 (6); 3398 (56); 3405 (14); 3411 (14); 3423 (56); 3527 (43); 3621 (47); 3767 (1); 3834 (30); 3868 (16); 4093 (46); 4114 (48); 4203 (43); 4465 (14); 4506 (43); 4668 (26); 4670 (19); 4675 (17); 4969 (16); 5255 (17); 5287 (8); 5299 (36); 5407 (42); 5574 (17); 5684 (39); 5960 (14); $6086(54) ; 6167(50)$; 6279 (25); 6325 (42); $6367(42) ; 6441$ (38).

Castroviejo: $9095 \mathrm{SC}(47)$.

Descoings: 12847 (4); 12848 (4).

Eneme: 361 (46); 362 (17); 374 (26); 378 (47); $386(50)$.

Eneme \& Lejoly: $37(44) ; 42(32) ; 68(26) ; 93$ (26); 132 (24); 141 (46).

Fernández Casas: 10230 (27); 11236 (16); 10373 (49); 11389 (50); 11442 (47); $11486(1)$; 11516 (16); 11567 (27); 11647 (47); 11711 (49); 11748 (27); 11775 (6); 11845 (49); 11846 (1); 11876 (27); 11916 (46); 11972 (23); 12035bis (47); 12045 (48); 12114 (16); 12122 (26); 12141 (6). 
TABLE 2

Species list of Melastomataceae in EQUatorial GuINEA, Distributions betweEN RÍ MUNI, BIOKO AND ANNOBÓN, NEW RECORDS

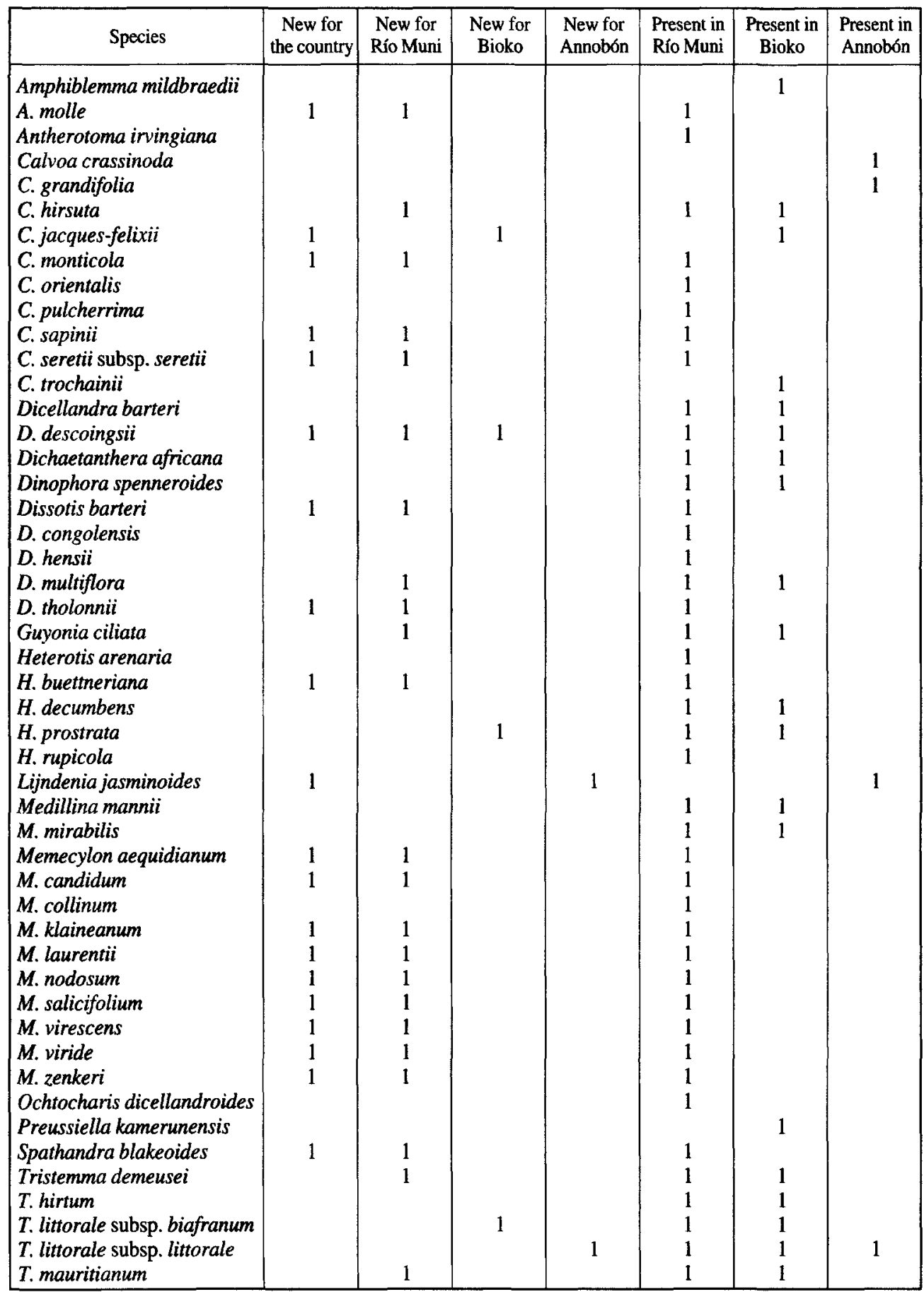


TABLE 2 (continued)

\begin{tabular}{|c|c|c|c|c|c|c|c|}
\hline Species & $\begin{array}{c}\text { New for } \\
\text { the country }\end{array}$ & $\begin{array}{l}\text { New for } \\
\text { Río Muni }\end{array}$ & $\begin{array}{c}\text { New for } \\
\text { Bioko }\end{array}$ & $\begin{array}{l}\text { New for } \\
\text { Annobón }\end{array}$ & $\begin{array}{l}\text { Present in } \\
\text { Río Muni }\end{array}$ & $\begin{array}{c}\text { Present in } \\
\text { Bioko }\end{array}$ & $\begin{array}{l}\text { Present in } \\
\text { Annobón }\end{array}$ \\
\hline $\begin{array}{l}\text { Tristemma oreophilum } \\
\text { T. rubens } \\
\text { Warneckea bebaiensis } \\
\text { W. cauliflora } \\
\text { W. fosteri } \\
\text { W. membranifolia } \\
\text { W. memecyloides } \\
\text { W. pulcherrima }\end{array}$ & $\begin{array}{l}1 \\
1 \\
1 \\
1 \\
1\end{array}$ & $\begin{array}{l}1 \\
1 \\
1 \\
1 \\
1\end{array}$ & 1 & 1 & $\begin{array}{l}1 \\
1 \\
1 \\
1 \\
1 \\
1 \\
1\end{array}$ & $\begin{array}{l}1 \\
1\end{array}$ & $\begin{array}{l}1 \\
1\end{array}$ \\
\hline TOTAL & 26 & 29 & 6 & 3 & 49 & 23 & 6 \\
\hline
\end{tabular}

GC: 11492 (13).

Guinea: 227-915 (24); 239-507 (14); 244-140 (7); $250(2) ; 252(17) ; 256(26) ; 256-689(46) ; 259$. 751 (46); 322 (26); 330 (26); 361 (14); 401 (17); 403 (17); 409 (46); 423 _(46); 463 (17); 482-123 (21); 483-122 (21); $493-118$ (16); 509 (2); 520 (14); 930bis (13); 935 bis (13); 981 (27); 1070 (27); 1189 (6); 1263 (47); 1265 (47); 1295 (27); 1319 (47); 1320 (47); $1347(27) ; 1387(27) ; 1396(27)$; $1723(1) ; 1839(49) ; 1902(49) ; 1968(1) ; 2079(6)$; 2297 (6); 2319 (14); 2321 (14); 2323 (14); 2434 (6); 2464 (26); 2514 bis (48).

Guinea \& Lope del Val: 229 (26); 663 (46); 976 (2); $977(2) ; 978(2) ; 979(21)$.

Lejoly: 93/312 (2); 93/333 (10); 93/350 (16); 94/117 (6); 94/123 (2); Lejoly 94/188 (8); 94/189 (6); 95T/L3.1023 (57); 95/139 (21); 95/146 (41); 95/174(31); 95/176(14); 97/013(21); 97/035(49); 97/036 (47); $97 / 088$ (17); 99/004 (22); 99/019 (45); $99 / 023(23) ; 99 / 028(27) ; 99 / 032$ (28); 99/036 (8); 99/048 (6); 99/066 (49); 99/081 (28); 99/082 (27); 99/083 (23); 99/088 (85); 99/100 (10); 99/102 (27); 99/134 (6); 99/155 (8); 99/156 (50); 99/185 (34); 99/196 (8); 99/202 (3); 99/231 (10); 99/238 (10); 99/268 (3); 99/276 (16); 99/310 (49); 99/314 (17); 99/331 (34); 99/349T1 (34); 99/350 (16); 99/364 (49); 99/365 (26); 99/367 (8); 99/368 (34); 99/373TI (16); 99/379 (8); 99/389 (50); 99/390 (23); 99/391 (45); 99/435 (22): 99/451.2 (49); 99/467bis (8); 99/492 (27); 01/04 (16); 01/20(14); 01/22 (6); 01/86 (21).

Lejoly \& Elad: 98/006 (21); 98/007 (17); 981036 (14); $98 / 077$ (18); $98 / 086$ (20); 981091 (27); 98/111 (26); 98/112 (24); $98 / 114$ (46); 98/124 (21); 98/184 (39); 98/201 (46); 98/202 (19).

Lejoly \& Esono: 49 (3); $57(55) ; 69(17) ; 75(8)$; 76 (27); 86 (34).

Lejoly \& Van Asbroeck: 11 (14); 35 (31).

Lisowski: 35 (44); 41 (46); 175 (47); 284 (44);
302 (26); 305 (47); 432 (47); 501 (47); 508 (46); $527(24) ; 549(46) ; 554(12) ; 562(47) ; 596(8) ; 663$ (3); $676(10) ; 697(14) ; 698(17) ; 729(21) ; 742$ (17); 757 (45); 787 (14); 797 (15); 813 (44); 881 (14); 911 (49); 948 (21); 1042 (21); 1043 (46); 1132 (47); 1209 (44); 1219 (26); 1322 (47); 1374 (20); 1374bis (21); 1385 (16); $1387(9) ; 1432(16)$; $1437(2) ; 1450(20) ; 1482(8) ; 1487(50) ; 1528(9)$; 1547 (14); $1580(19)$.

Lope de Val: s.n. (MA 500350) (27); s.n. (MA 500350) (46).

Mildbraed: 6528(4).

Ngomo: 75 (17); 94 (26); 241 (14); 321 (21); $356(20) ; 423$ (39).

Nguema: 86 (15); 135 (14); 187 (42); 434 (6); 465 (2); 1064 (26); $1167(46)$.

Nguema \& Esono: 286 (49); 292 (2).

Nguema, Esono \& Lejoly: 278 (15); 279 (6).

Nguema \& Lejoly: 5 (47); 9 (14); $156(50) ; 185$ (6); $196(6) ; 198(51)$.

Nguema \& Parmentier: 509 (32).

Obama: 159 (49); $180(17) ; 305$ (47); $306(17)$; $346(36) ; 350(6) ; 733(26) ; 889(44)$.

Obama \& Lejoly: 355 (20); 356 (21); 358 (17); 367 (10); 375 (34); 409 (14), 449 (21); 455 (17); 475 (46); $480(3) ; 535(8) ; 628(3) ; 683$ (17).

Parmentier: 1510 (28); 1512 (23); 1515 (45); 1530 (22); 1561 (10); 1574 bis (3); $1613(8) ; 1695$ (17); 3039 (45).

Parmentier \& Esono: 2721 (22); 2727 (27); 2937 (45); 2739 (45); 2763 (22); 2798 (30); 2806 (6); 2836 (8); 2861 (2); $2867(15) ; 2875$ (14); 2877 (2): 2907 (50); 2974 (49); 2983 (28); 2893 (6); 2905 (8); 2906 (21); 2916 (15); 2964 (11); 2965 (6): 2972 (45); 2982 (10); 3097 (27); 3098 (3); 3150 (27); 3151 (17); 3152 (21); $3214(57) ; 3316$ (15); 3340 (8); 3378 (26); 3388 (17); 3394bis (30); 3402 (10); 3410 (42); $3412(2) ; 3431(6), 3432(6)$; 3448 (15); 3453 (22); 3490 (10); 3491 (3); 3495 
(18); 3500 (34); $3518(8) ; 3519(34) ; 3544$ (18); 3560 (55); $3578(20) ; 3579(49) ; 3580(50) ; 3581$ (17); 3582 (26); 3596 (10); 3600 (8); 3622 (3); 3625 (16); 3640 (49); 3674 (17); 3697 (3); 3716 (10); 3796 (34); 3797 (8); 3827 (17); 3828 (49); $3829(20)$.

Parmentier \& Nguema: 294 (10); 301 (10); 375 (8).

Pérez Viso: 3308 (49); 3369 (21); 3459 (49); 3450 (17); 3483 (17); 3507 (26); 3541 (46); 3647 (14); 3756 (17).

Porembski, Biedienger \& Nguema: 3503 (10); 3599 (3).

Senterre \& Ngoma: $15(55) ; 16(14) ; 25(55) ; 37$ (45); 38(17); 267 (14); $290(50) ; 483(10) ; 799(8)$; 801 (35); 805 (35); $2167(8) ; 3419(41)$.

Senterre \& Obiang: 3305 (37); 3604 (53); 3649 (52); 3822 (6).

Senterre, Obiang \& Esono: $2817(36) ; 2848(36)$.

Senterre, Obiang \& Ngomo: $2068(50) ; 2123$ (31); 2364 (57).

Senterre, Obiang \& Nguema: 2153 (2).

Stevart: 913 (33); 995 (10); 1002 (8); $1010(30)$. Van Reeth: 43 (6); 72 (45); 140 (2); 334 (40). Velayos, Aedo \& Pérez-Viso: 9524 (14).

Wrigley: 65 (48); 201 (54);

Wrigley \& Melville: 164 (5).

\section{ACKNOWLEDGMENTS}

We are grateful to the Biod-iberia project that allowed us to work on the MA material, and to all the staff of MA, especially to Mauricio Velayos, Francisco Cabezas, Ramón Morales and Carlos Aedo. We thank the WAG herbarium for the loan of material. We thank BR for use of the African Herbarium. Estrella Figueiredo determined part of the Calvoa specimens. We are grateful to JeanClaude Moniquet and Nicolas Barbier (Université Libre de Bruxelles, BRLU) for their valuable help. This work was partially financied by the Spanish Dirección General de Investigación Científica y Técnica (DGICYT) through the research project REN 2002-011299.

\section{REFERENCES}

Aedo, C., M.T. Tellería \& M. Velayos (1999). Bases documentales para la Flora de Guinea Ecuatorial. Plantas vasculares y hongos. CSIC, Madrid: $414 \mathrm{pp}$.

BORHIDI, A. (1993). Warneckea hedbergorum sp. n. (Memecylaceae) and a short review of the East African Memecylon s.l. Op. Bot. 121: 149-151.

BRENAN, J.P.M. (1979). Some aspects of the phytogeography of Tropical Africa. Ann. Missouri Bot. Gard. 65(2): 437-478.
Fernández Casas, F.J. \& R. Morales Valverde (1995). Proyecto de una flora de la isla de Bioko (Guinea Ecuatorial) . Anales Jard. Bot. Madrid 52 (2): 230240.

Figueiredo, E. (2001). A revision of Calvoa Hook. $f$. (Melastomataceae). Bot. J.Linn. 136(2): 179-205.

GUINEA LÓPEZ, E. (1946). Ensayo geobotánico de la Guinea Continental Española. Dirección General de Maruecos y Colonias. Madrid: 388 p.

GUINEA LOPEZ, E. (1951). Melastomataceae Fernandopoanae Bol. Real Soc. Esp. Hist. Nat., Secc. Biol. 49(3): 175-187.

HEDBERG. I. \& O. HEDBERg (1968). Conservation of vegetation in Africa South of the Sahara. Acta Phytogeogr. Suec. 54(XI): 1-320.

Hutchinson, J. (1928). Flora of West Tropical Africa, vol. 1(2). The Crown agents for the colonies 1(2): 247-523.

Hutchinson, J. \& J.M. Dalziel (1954). Flora of West Tropical Africa, 2nd. edition, vol. 1(1). The Crown agents for Oversea Governments and Administrations 1(1): 1-295.

JACQUES-FÉliX, H. (1974a). Le genre Amphiblemma Naud. (Melastomacées). Adansonia 13(4): 429-459.

JACQUES-FÉl.IX, H. (1974b). Le genre Dicellandra Hook. f. (Melastomacées). Adansonia 14(1): 77-98.

JACQUES-FÉlIX, H. (1976). Le genre Tristemma Jussieu (Melastomataceae). Bull. Mus. Nation. Hist. Nat. Sér. 3(418), Bot. 28: 137-207.

JACQUES-FÉLIX, H. (1977). Synonymes nouveaux de Melastomatacées d'Afrique et de Madagascar (New synonyms of some Melastomataceae from Africa and Madagascar). Adansonia 17(1): 77-78.

JACQUES-FÉLIX, H. (1983a). Flore du Cameroun: 24. Melastomatacées. Yaounde, Délégation Generale a la Recherche Scientifique et Technique: 192 pp.

JACQUES-FÉlXX, H. (1983b). Flore du Gabon: 25. Melastomatacées. Paris, Museum National d'Histoire Naturelle: $167 \mathrm{pp}$.

JACQUES-FÉLIX, H. (1987). Un nouvel Heterotis (Melastomataceae) de Gabon. Bull. Mus. Nation. Hist. Nat. . B. Adansonia 9(3): 255-257.

JOHANSSON, D. (1974). Ecology of vascular epiphytes in West African rain forest. Acta Phytogeogr. Suec. 59: 1-129.

LEBRUN. J.P. \& A.L. STORK (1991). Enumération des plantes à fleur d'Afrique tropicale, Vol. I: Généralités et Annonaceae à Pandaceae. Éditions des Conservatoire et Jardin Botaniques de la Ville de Genève: $249 \mathrm{pp}$.

LEBRUN, J.P. \& A.L. STORK (1995). Enumération des plantes à fleur d'Afrique tropicale. Vol. III: Monocotylédones: Limnocharitaeae à Poaceae. Éditions des Conservatoire et Jardin Botaniques de la Ville de Genève: 341 pp.

Lebrun, J.P. \& A.L. STORK (1997). Enumération des plantes à fleur d'Afrique tropicale. Vol. $N$ : Gamopétales: Clethraceae à Lamiaceae. Éditions des Conservatoire et Jardin Botaniques de la Ville de Genève: $712 \mathrm{pp}$.

LEJOLY, J. \& S. LISOWSKI (1999a). Novitates Guinea Aequatorialis (1). Les plantes des sables côtiers et des 
prairies Litorales de la Région continentale (Río Muni). Bull. Jard. Bot. Nat. Belg. 67: 99-106.

LEJOLY, J. \& S. LISOWSKI (1999b). Novitates Guineae Aequatorialis (5). Premier aperçu sur la végétation des inselbergs au Río Muni. Bull. Jard. Bot. Nat. Belg. 67: 114-121.

LEJOLY, J. \& S. LISOWSKI (1999c). Novitates Guineae Aequatorialis (6). Heterotis obamae (Melastomataceae) espece nouvelle du Río Muni. Syst. Geogr. Pl. 69(2): 185-188.

MILDBRAED, J. (1922). Wissenschaftliche Ergebnisse der Zweiten Deutschen Zentral-Afrika-Expedition 19101911. Band II: BotanikVerlag von Klinkhardt \& Biermann (Hamburgische Weissenschaftliche Stiftung) 2: 146-195.

MORALES, R. (1994). Melastomataceae Guineae Aequatorialis nonnullac. Fontqueria 40: 13-18.

Morales, R. (1995). Melastomataceae Guineae Aequatorialis nonnullae, II. Fontqueria 42: 17-18.

ParmentIER, I. (2002). Premières études sur la diversité végétale des inselbergs de Guinée Equatoriale continentale. Proceedings du XVI Congrès de l'AETFAT. Syst. Geog. Pl. 71(2): 911-922.

Parmentier, I., J. Lejoly \& N. Nguema (2001). La végétation des inselbergs du monument naturel de Piedra Nzas (Guinée Equatoriale continentale). Acta Botanica Gallica 148(4): 341-365.

PARMENTIER, I. \& N. NGUEMa (2001). La vegetación de los inselbergs de Río Muni. In: C. Aedo, R. Morales. M.T. Tellería \& M. Velayos (eds.), Botánica y botánicos en Guinea Ecuatorial. Real Jardín Botánico-
Agencia Española de Cooperación Internacional. Madrid: 201-230.

Pérez Del VAL, J. (1996). Las aves de Bioko, Guinea Ecuatorial. Edilesa. León.

SENTERRE. B. (2001). La phytogéographie des forêts denses de Guinée Equatoriale, avec une attention particulière aux forêts de Nsork. Proceedings of the XVI Congres de l'AETFAT. Syst. Geogr. Pl. 71: 10871096.

SeNTERRE, B. \& J. Lejoly (2001). Los grandes tipos de hábitat forestales de Río Muni. In: C. Aedo, R. Morales, M.T. Tellería \& M. Velayos (eds.), Botánica y botánicos en Guinea Ecuatorial. Real Jardín BotánicoAgencia Española de Cooperación Internacional. Madrid: $171-199$.

SenterRe. B. \& N. NGUEma (2001), La diversité des ligneux dans la forêt de Nsork (Guinée Equatoriale). Proceedings of the XVI' Congrès de l'AETFAT. Syst. Geogr. Pl. 71: 837-846.

VELAYOS, M. \& C. AEDO (2001). Exploraciones botánicas en Guinea Ecuatorial. In: C. Aedo, R. Morales, M.T. Tellería \& M. Velayos (eds.), Botánica y botánicos en Guinea Ecuatorial. Real Jardín BotánicoAgencia Española de Cooperación Internacional. Madrid: 23-49.

Vel.AYOS, M.. C. AEdo \& R. PÉrez VISO (2001). Checklist of the pteridophytes of Equatorial Guinea. Belg. Journ. Bot. 134(2): 145-191.

Editado por Mauricio Velayos Aceptado para publicación: 10-VI-2003 